\title{
Analysis of the silicon solar cells efficiency. Type of doping and level optimization
}

\author{
A.V. Sachenko ${ }^{1}$, V.P. Kostylyov ${ }^{1}$, M.V. Gerasymenko ${ }^{2}$, R.M. Korkishko ${ }^{1}$, M.R. Kulish ${ }^{1}$, M.I. Slipchenko ${ }^{2}$, \\ I.O. Sokolovskyi ${ }^{1^{*}}$, V.V. Chernenko ${ }^{1}$ \\ ${ }^{I} V$. Lashkaryov Institute of Semiconductor Physics, NAS of Ukraine \\ 41, prospect Nauky, 03028 Kyiv, Ukraine \\ ${ }^{2}$ Kharkiv National University of Radio Electronics \\ 14, Lenin ave., 61166 Kharkiv, Ukraine \\ "Corresponding authore-mail addresses: sach@isp.kiev.ua (A.V.Sachenko),vkost@isp.kiev.ua (V.P.Kostylyov), \\ n.v.gerasimenko@mail.ru (M.V.Gerasymenko),romkin.ua@gmail.com (R.M. Korkishko), \\ n_kulish@yahoo.com (M.R.Kulish),nslip@kture.kharkov.ua (M.I.Slipchenko), \\ i.o.sokolovskyi@gmail.com (I.O.Sokolovskyi),vvch@isp.kiev.ua (V.V.Chernenko)
}

\begin{abstract}
The theoretical analysis of photovoltaic conversion efficiency of highly effective silicon solar cells (SC) has been performed for $n$-type and $p$-type bases. Considered here is the case when the Shockley-Read-Hall recombination in the silicon bulk is determined by the deep level of Fe. It has shown that, due to asymmetry of recombination parameters inherent to this level, the photovoltaic conversion efficiency is increased in SC with the $n$-type base and decreased in SC with the $p$-type base with the increase in doping. Two approximations for the band-to-band Auger recombination lifetime dependence on the base doping level are considered when performing the analysis. The experimental results are presented for the key characteristics of SC based on a-Si:H- $n$-Si heterojunctions with intrinsic thin layer (HIT). A comparison between the experimental and calculated values of the HIT cell characteristics has been made. The surface recombination velocity and series resistance are determined from it with a complete coincidence of the experimental and calculated SC parameters' values. Apart from the key characteristics of SC, surface recombination rate and series resistance were determined from the results of this comparison, in full agreement with the experimental findings.
\end{abstract}

Keywords: silicon solar cell, heterojunction, doping level, Shockley-Read-Hall recombination, Auger recombination.

Manuscript received 12.11.15; revised version received 26.01.16; accepted for publication 16.03.16; published online 08.04.16.

\section{Introduction}

When analyzing the dependence of the silicon solar cells (SC) on the base doping level, the assumption is commonly used that the deep levels close in $E_{t}$ energy to the middle of the band gap with close electron- and holecapture cross-sections are responsible for the ShockleyRead-Hall (SRH) recombination. Specifically, the Au level meets these criteria (Hangleiter, 1987). At the same time, the SRH recombination can be determined by the energy levels which do not agree with the middle of the band gap and electrons $\sigma_{n}$ and holes $\sigma_{p}$ cross-sections differ essentially. The level of Fe is one of such levels $[2,3]$. According to [2], it is characterized by the values $E_{c}-E_{t}=0.774 \mathrm{eV}\left(E_{c}\right.$ is the conduction band edge $), \sigma_{n}=$ $5 \cdot 10^{-14} \mathrm{~cm}^{2}, \sigma_{p}=7 \cdot 10^{-17} \mathrm{~cm}^{2}$. In this case, the SRH lifetime value $\tau_{\mathrm{SRH}}$ can significantly depend both on the SC base doping and on the excess electron-hole pairs' 
concentration $\Delta n$ in the base. These dependences can significantly affect the minority-carriers effective bulk lifetime $\tau_{\text {eff }}$ determined by the relation of different recombination mechanisms, including the $\mathrm{SRH}$ recombination, radiative recombination and band-toband Auger recombination.

The analysis of the photovoltaic conversion efficiency $\eta$ is carried out in this research for highly effective silicon SC and HIT $^{1}$ (heterojunction with intrinsic thin layer) SC, depending on the base type and doping level for the case when the SRH recombination lifetime is determined by the Fe level. Calculation results are compared to the experimental values of the photovoltaic conversion efficiency $\eta$, open-circuit voltage $V_{O C}$, fill-factor $F F$ and some other parameters of $\mathrm{SC}$ based on a-Si:H- $n$-Si SC.

A simple approach is used in the analysis, which makes it possible to model characteristics of SC produced on the crystalline base [4]. Its special feature is the fact that one of the main characteristics of SC is the short-circuit current density $J_{S C}$ is found experimentally and the remaining SC parameters are calculated. This essentially simplifies the analysis of the experimental results, which can serve as a basis for optimization of such characteristics of the HIT cells as the doping level of the base $N_{d}\left(N_{a}\right)$ under the given SRH lifetime $\tau_{\mathrm{SRH}}$ value, surface recombination velocity $S_{0}$ and $S_{d}$ on the $\mathrm{SC}$ frontal and rear surfaces and series resistance $R_{S}$.

\section{Analysis of $\tau_{\mathrm{SRH}}$ dependence on the base doping level for the cases of the base area of $p$ - and $n$-type}

Using the approach developed in [4], let us write the expressions for $\tau_{\mathrm{SRH}}$ value, when the recombination time determines the level of Fe for the SC base of $p$ - and $n$ type, respectively [5]:

$$
\begin{gathered}
\tau_{S R H}^{p} \equiv \frac{\tau_{p 0}\left(n_{1}+\Delta n\right)+\tau_{n 0}\left(N_{a}+p_{1}+\Delta n\right)}{\left(N_{a}+\Delta n\right)}, \\
\tau_{S R H}^{n} \cong \frac{\tau_{p 0}\left(N_{d}+n_{1}+\Delta n\right)+\tau_{n 0}\left(p_{1}+\Delta n\right)}{\left(N_{d}+\Delta n\right)},
\end{gathered}
$$

where $\tau_{S R H}^{p}$ and $\tau_{S R H}^{n}$ are the SRH lifetimes for the $p$ and $\quad n$-type bases, $\quad \tau_{p 0}=\left(1.19 \cdot 10^{-9} N_{t}\right)^{-1} \mathrm{~s}$, $\tau_{n 0}=\left(8.5 \cdot 10^{-7} N_{t}\right)^{-1} \mathrm{~s}, n_{1}$ and $p_{1}$ are concentrations of electrons and holes for the cases when the position of the recombination level coincides with the Fermi level, and $n_{1}=n_{i}(T) \exp (-8.327), p_{1}=n_{i}(T) \exp (8.327), n_{i}(T)$ is the intrinsic carriers concentration in silicon, $T=25^{\circ} \mathrm{C}$. Numerical parameters for $\tau_{p 0}, \tau_{n 0}, n_{1}$ and $p_{1}$ for calculation are taken from [2].

We note that, in the expressions (1) and (2), it is implicitly assumed that the excess densities of electrons and holes are equal. This condition holds, if these

${ }^{1}$ HIT (heterojunction with intrinsic thin layer) is a trademark of Panasonic Group densities notably exceed the concentration of recombination levels. In the case considered here, this is indeed the case, as will be discussed below.

Hereinafter, the case of Si with high effective bulk lifetime values will be considered, when the minority charge carriers diffusion length is significantly higher than the SC base thickness $d$ throughout the doping level range, i.e. the effective diffusion length $L_{\text {eff }}=$ $\left(D_{n(p)} \tau_{e f f}^{n(p)}\right)^{1 / 2}>>d$. Here $D_{n(p)}$ and $\tau_{e f f}^{n(p)}$ are the diffusion coefficient and effective lifetime for electrons (holes). In this case, the excess minority charge carriers concentration $\Delta n$ is constant along the base.

Fig. 1 demonstrates dependences of $\tau_{S R H}^{p}$ and $\tau_{S R H}^{n}$ on the base doping level for two $\Delta n$ values: $5 \cdot 10^{14}$ and $5 \cdot 10^{15} \mathrm{~cm}^{-3}$. Note that the first value for the base doping levels less or equal to $10^{15} \mathrm{~cm}^{-3}$ is typical for the silicon SC operating at AM1.5 under the maximum power take-off conditions, and the second value is typical for the case of the silicon SC operating in the open circuit mode. When calculating the $\tau_{\mathrm{SRH}}$ value, the $N_{t}$ value was supposed to be equal to $6 \cdot 10^{11} \mathrm{~cm}^{-3}$. Thus, the inequality $\Delta n>>N_{t}$ holds well for SC considered here. Fig. 1 shows that the $\tau_{S R H}^{p}$ value decreases strongly (more than two orders of magnitude) with the base doping level increase, and strongly depends on the $\Delta n$ value. At the same time, the $\tau_{S R H}^{n}$ value practically does not depend either on the base doping or on the $\Delta n$ value. With the concentration of the iron recombination centres equal to $6 \cdot 10^{11} \mathrm{~cm}^{-3}$ chosen for calculations, it is equal to $\tau_{S R H}^{n}=1.4 \mathrm{~ms}$ and concides with the measured value of the bulk lifetime in the $n$-type material with the doping level $1.6 \cdot 10^{15} \mathrm{~cm}^{-3}$. Such a lifetime is typical for high quality silicon.

This result is close to that obtained in the work [6], which shows that the SRH lifetime as a function of the doping level is practically constant at $N_{d} \geq 10^{15} \mathrm{~cm}^{-3}$.

\section{Fundamental relations determining the silicon $\mathrm{SC}$ efficiency}

Using the approach developed in [4], let us write the relations determining the photovoltaic conversion efficiency of the highly effective silicon SC and HIT SC $\eta$. Let us note in the beginning that, in addition to the $L_{\text {eff }} \gg d$ inequality, the $\Delta n_{O C} \geq N_{d}\left(N_{a}\right)$ condition is usually implemented, where $\Delta n_{O C}$ is the excess electron-hole pairs concentration in the base for the open circuit case. So, the expressions for the open circuit voltage $V_{O C}$ in the $p$ - and $n$-type base cases can be written [7], respectively, as

$$
V_{O C} \cong \frac{k T}{q} \ln \left(\frac{\Delta n_{O C}}{p_{0}}\right)+\frac{k T}{q} \ln \left(1+\frac{\Delta n_{O C}}{N_{d}}\right),
$$


$V_{O C} \cong \frac{k T}{q} \ln \left(\frac{\Delta n_{O C}}{n_{0}}\right)+\frac{k T}{q} \ln \left(1+\frac{\Delta n_{O C}}{N_{a}}\right)$,

where $k$ is the Boltzmann constant, $T$ - SC temperature, $q$ - elementary charge, $p_{0}=n_{i}^{2}(T) / N_{d}$ - equilibrium concentration of holes in the $n$-type base, and $n_{0}=n_{i}^{2}(T) / N_{a} \quad-$ equilibrium concentration of electrons in the $p$-type base.

For $\Delta n_{O C} \geq N_{d}\left(N_{a}\right)$, the open-circuit voltage $V_{O C}$ is higher than $V_{O C}$ for the standard case, when $\Delta n_{O C}<N_{d}\left(N_{a}\right)$.

The generation-recombination balance equation in the case of the open-circuit mode, when implementing inequality $L_{e f f}>d$, can be written as

$I_{S C} / q=A_{S C}\left[\frac{d}{\tau_{b}}+S\right] \Delta n_{O C}$

Here $I_{S C}$ is the short-circuit current, $A_{S C}$ - surface area of $\mathrm{SC}, \tau_{b}=\left[\tau_{\mathrm{SRH}}^{-1}\left(\Delta n_{O C}\right)+A\left(N_{d}\left(N_{a}\right)+\Delta n_{O C}\right)+R_{\text {Auger }}\right]^{-1}$ is the bulk lifetime, $A \approx 6.3 \cdot 10^{-15} \mathrm{~cm}^{3} / \mathrm{s}$ [8] is the radiative recombination coefficient in silicon, $S=$ $S_{0}+S_{d}$. The rate (inverse time) of the band-to-band Auger recombination $\left(R_{\text {Auger }}\right)$ in the $n$-type silicon is determined using the expression

$R_{\text {Auger }}=C_{n}\left(N_{d}+\Delta n_{O C}\right)^{2}+C_{p}\left(N_{d}+\Delta n_{O C}\right) \Delta n_{O C}$,

where $\quad C_{n}=\left(2.8 \cdot 10^{-31}+\frac{2.5 \cdot 10^{-22}}{\left(N_{d}+\Delta n_{O C}\right)^{0.5}}\right) \mathrm{cm}^{6} / \mathrm{s}$, $C_{p}=10^{-31} \mathrm{~cm}^{6} / \mathrm{s}[9,10]$. The second term in the expression for $C_{n}$ takes into account the many-electron effects, namely the effect of spatial correlation for distribution of two electrons and one hole involved in the act of Auger recombination, conditioned by the Coulomb interaction [9].

In the $p$-type silicon

$R_{\text {Auger }} \equiv C_{p}\left(N_{a}+\Delta n_{O C}\right)^{2}+C_{n}\left(N_{a}+\Delta n_{O C}\right) \Delta n_{O C} \quad$ and

$C_{n}=\left(2.8 \cdot 10^{-31}+\frac{2.5 \cdot 10^{-22}}{\Delta n_{O C} 0.5}\right) \mathrm{cm}^{6} / \mathrm{s}$.

Note that the expressions (6) and (7) for $C_{n}$ are approximate. A choice of this expression was discussed in more details in [10]. It was demonstrated that various dependences were obtained in different experiments. The analysis of numerous experimental data, obtained in the case of silicon, makes it possible to derive the empirical expression fully describing the dependence of $R_{\text {Auger }}$ on the equilibrium concentrations of electrons $n_{0}$ and holes $p_{0}$ in the SC base, as well as on the concentration of excess electron-hole pairs $\Delta n$ at $T=300 \mathrm{~K}$ [11]:

$R_{\text {Auger }}=\frac{\left(n_{0}+\Delta n\right)\left(p_{0}+\Delta n\right)\left(1.8 \cdot 10^{-24} n_{0}^{0.65}+6 \cdot 10^{-25} p_{0}^{0.65}+3 \cdot 10^{-27} \Delta n^{0.8}+9.5 \cdot 10^{-15}\right)}{\Delta n}$. 


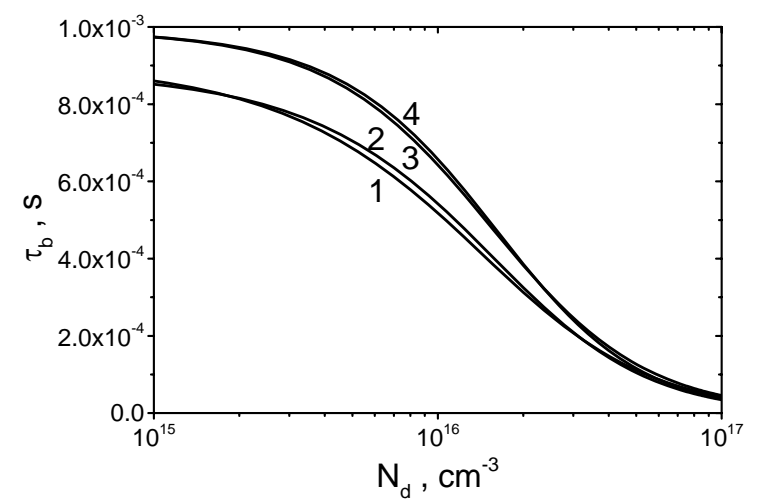

Fig. 2. Dependence of the effective lifetime in the bulk base on the $n$-type base doping level of SC obtained using (8) (curves 1 and 3) and (9) (curves 2 and 4). The parameters used are: $\tau_{\mathrm{SR}}=$ $1.4 \mathrm{~ms}, I_{S C}=142.7 \mathrm{~mA}, S=12 \mathrm{~cm} / \mathrm{s}$ (curves 1 and 2 ) and $1 \mathrm{~cm} / \mathrm{s}$ (curves 3 and 4 ).

To calculate the photovoltaic conversion efficiency, a theoretical expression for SC current-voltage characteristic is needed. With this aim in view, we proceed as follows. Let us replace $V_{O C}$ in (10) by the applied forward bias $V$ value. Besides, it is also necessary to take into account the voltage drop on the series resistance $R_{s}$. This operation makes it possible to determine $\Delta n(V)$ :

$$
\begin{aligned}
& \Delta n(V)=-\frac{N_{d}\left(N_{a}\right)}{2}+ \\
& +\sqrt{\frac{\left(N_{d}\left(N_{a}\right)\right)^{2}}{4}+n_{i}^{2}\left(\exp \frac{q\left(V+I R_{s}\right)}{k T}-1\right)}
\end{aligned}
$$

where $I$ is the current.

In what follows, let us generalize equation (5), correct for the open circuit case, for the $V<V_{O C}$ case, i.e. for nonzero current. Then, this equation can be written as:

$I(V)=I_{S C}-I_{r e c}(V)$,

where

$$
I_{\text {rec }}(V)=q A_{S C}\left(\frac{d}{\tau_{b}}+S\right) \Delta n(V),
$$

and the $\tau_{\mathrm{b}}$ value can be found using (5) (with the replacement of $\Delta n_{O C}$ by $\Delta n(V)$.

The $V_{m}$ value is found from the maximum power take-off condition $d(V J(V)) / d V=0$, and its substitution in (13) makes it possible to determine the value of $I_{m}$. As a result, we obtain the photoconversion power:

$$
P=I_{m} V_{m} \text {. }
$$

For the fill-factor, the standard expression can be used:

$$
F F=\frac{I_{m} V_{m}}{I_{S C} V_{O C}} .
$$

The photoconversion efficiency is also given by the known formula:

$$
\eta=\frac{I_{m} V_{m}}{A_{S C} P_{S}},
$$

where $P_{S}$ is the incident solar radiation power density.

\section{Comparative analysis of the obtained relations for the SC base of $n$ - and $p$-types}

When building theoretical dependences for characteristics of silicon SC with the base of the $n$-type, the expressions for $R_{\text {Auger }}$ defined both by the relation (9) and the expression (8) were used. The following figures were built using (9) and (8) for SC with the $n$-type base and (5), (7) in the case of SC with $p$-type base.

Fig. 3 demonstrates the theoretical dependence for the open-circuit voltage on the base doping level for the $p$ - and $n$-type bases. The same parameters values determining the SRH lifetime were used for plotting the curves in Figs. 3 and 1. The values of the surface recombination rate $S$ and short-circuit current density $J_{S C}$ varied when calculating the curves 1-4. As Fig. 3 suggests, the $V_{O C}$ values coincide both for $n$ - and $p$-type bases when the doping levels are low $\left(\leq 10^{15} \mathrm{~cm}^{-3}\right)$ (compare 1,2 and 3, 4 curves calculated with the same $S$ and $J_{S C}$ values). It should be noted that $V_{O C}$ values do not depend on the doping level, if $\Delta n_{0}>N_{d}\left(N_{a}\right)$ inequality is satisfied. With $N_{d} \geq 10^{15} \mathrm{~cm}^{-3}$, the $V_{O C}$ values in $\mathrm{SC}$ with the $n$-type base increase, subsequently they reach a maximum and begin to decrease. In this case, the $V_{O C}$ value decline is caused by the prevalence of the Auger band-to-band recombination.

The $V_{O C}\left(N_{a}\right)$ dependences in SC with $p$-type base behave quite differently. With $N_{a} \geq 10^{14} \mathrm{~cm}^{-3}$ the $V_{O C}$ values initially decrease slowly, and with $N_{a}>5 \cdot 10^{15} \mathrm{~cm}^{-3}$ the rate of $V_{O C}$ decline increases significantly. In this case, the $V_{O C}\left(N_{a}\right)$ decline is associated with the SRH lifetime decrease. Strictly speaking, in the framework of the used approximations the calculated dependences $V_{O C}\left(N_{a}\right)$ will be sufficiently accurate, with the inaccuracy not exceeding $2 \%$, only with the fulfilment of the $L>2 d$ condition, which is implemented with $N_{a}<9 \cdot 10^{15} \mathrm{~cm}^{-3}$.

At higher doping levels, not all of electron-hole pairs generated in the $\mathrm{SC}$ base will reach the $p$ - $n$ junction, which will primarily result in the short-circuit current $I_{S C}$ reduction due to the reduction of the current collection coefficient. In our calculations, we use the experimental value of the short-circuit current and, furthermore, we consider it as independent of the doping level. Therefore, the inequality $L \geq 2 d$ is also necessary so that the quantum yield $I_{S C}$ does not decrease with increasing the doping level. 


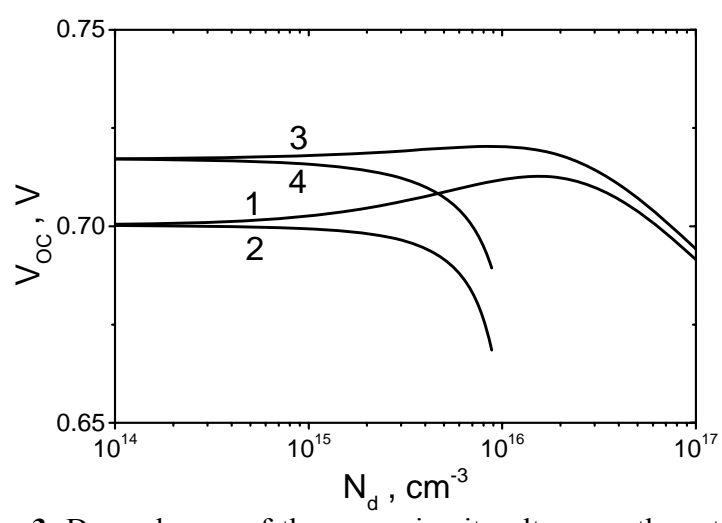

Fig. 3. Dependences of the open-circuit voltage on the $n$-type base doping level (curves 1 and 3) and $p$-type base doping level (curves 2 and 4). Parameters used for calculation: $d=$ $300 \mu \mathrm{m}, \tau_{\mathrm{SR}}=1.4 \mathrm{~ms}, T=298 \mathrm{~K}$. Curves 1 and 3 calculated with $S=12 \mathrm{~cm} / \mathrm{s}, J_{S C}=36 \mathrm{~mA} / \mathrm{cm}^{2}$ and curves 2 and 4 calculated with $S=1 \mathrm{~cm} / \mathrm{s}, J_{S C}=39.5 \mathrm{~mA} / \mathrm{cm}^{2}$.



Fig. 4. Dependence of voltage on the base doping level in the mode of the maximum power take off: curves 1 and 3 correspond to the $n$-type base, curves 2 and 4 correspond to the $p$-type base. $R_{S}=0.21 \mathrm{Ohm}$.

Fig. 4 demonstrates the theoretical dependences of voltage $V_{m}$ on the $p$ - and $n$-type base doping level in the mode of the maximum power take off. The same parameters values were used for construction of Fig. 4 as for Fig. 3.

As it was evidenced by the comparison between Figs. 3 and 4, behavior of $V_{O C}$ and $V_{m}$ depending on the base doping level is very similar in both cases with the only difference that for the dependences $V_{m}\left(N_{d}\left(N_{a}\right)\right)$ the $V_{m}$ value region of independence on $N_{d}\left(N_{a}\right)$ and the point of the $V_{m}\left(N_{a}\right)$ minimum are shifted to the region of lower doping levels' values. Thus, the region of independence of $V_{m}$ on $N_{d}\left(N_{a}\right)$ is realized when $N_{d}\left(N_{a}\right) \leq 10^{14} \mathrm{~cm}^{-3}$, and the point of the $V_{m}$ minimum is realized when $N_{a} \approx 10^{15} \mathrm{~cm}^{-3}$. It should be noted that, in the case of $p$-type base, the $L>2 d$ condition, necessary for the $V_{m}$ correct calculation, is fulfilled if $N_{a} \leq 10^{15} \mathrm{~cm}^{-3}$.
Fig. 5 demonstrates the theoretical dependences of the photovoltaic conversion efficiency $\eta$ on the doping level of the $p$ - and $n$-type bases. The same parameter values were used for construction of Fig. 5 as for Figs. 3 and 4. As Fig. 5 suggests, the dependences of photovoltaic conversion efficiency $\eta$ on the base doping level repeated the dependence $V_{m}$ on $N_{d}\left(N_{a}\right)$ (see Fig. 4) with a certain scaling. The essential difference of these dependences for SC with the base of the $n$ - and $p$-types is that in SC with the $n$-type base the $\eta$ values grow with the growth of the base doping level and in solar cells with $p$-type base the $\eta$ values decrease. As already mentioned above, the decrease in $\eta\left(N_{a}\right)$ with the increase in $N_{a}$ in SC with the $p$-type base is associated with a decrease in the SRH lifetime. Fig. 5 shows that, for the typical doping levels of SC bases $(1 \ldots 4) \cdot 10^{15} \mathrm{~cm}^{-3}$, the efficiency of SC with the $n$-type base significantly exceeds the efficiency of SC with the $p$-type base. The increase in the $\eta$ value in SC with the $p$-type base can be achieved by reducing the concentration of the recombination centres of iron.

Fig. 6 shows the calculated dependence of the photovoltaic efficiency $\eta$ on the base doping level for the $n$ - and $p$-type bases for the case where only the base thickness varies. The calculation was performed for the case when the base thickness $d$ was equal to 100,200 and $300 \mu \mathrm{m}$. As can be seen from Fig. 6, the lower the thickness of the base, the greater the efficiency $\eta$. This increase is associated with the increase in the opencircuit voltage $V_{O C}$, more precisely, in the voltage in the mode of the maximum power take off $V_{m}$ due to the bulk recombination reduction.

The doping levels' values, with which the dependences $\eta\left(N_{a}\right)$ are broken in the case of SC with the $p$-type base, correspond to the $L_{\text {eff }}\left(N_{a}\right) \approx 2 d$ conditions. As Fig. 6 suggests, the lower the base thickness $d$, the higher doping levels required for the $L_{\text {eff }}\left(N_{a}\right) \geq 2 d$ condition fulfilment.

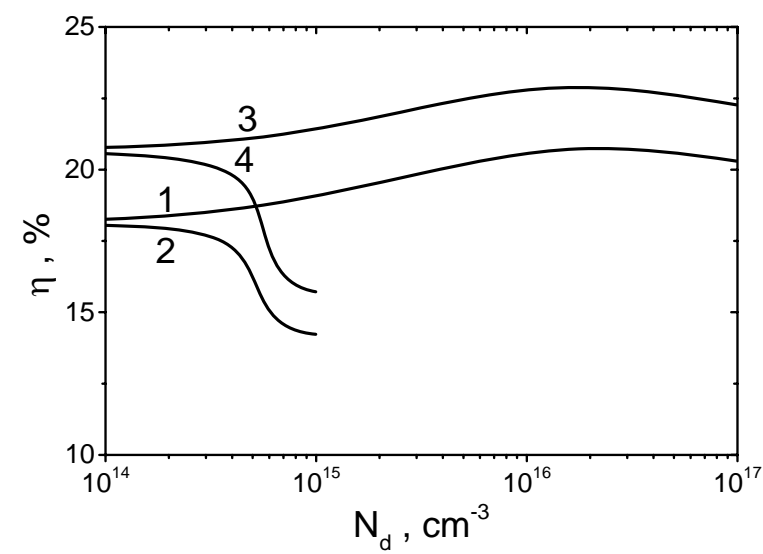

Fig. 5. Dependence of the photovoltaic conversion efficiency on the doping level base: curves 1 and 3 correspond to the $n$-type base, and curves 2 and 4 correspond to the $p$-type base. The same parameter values were used for construction of Fig. 5 as for Fig. 3. $R_{S}=0.21 \mathrm{Ohm}$. 


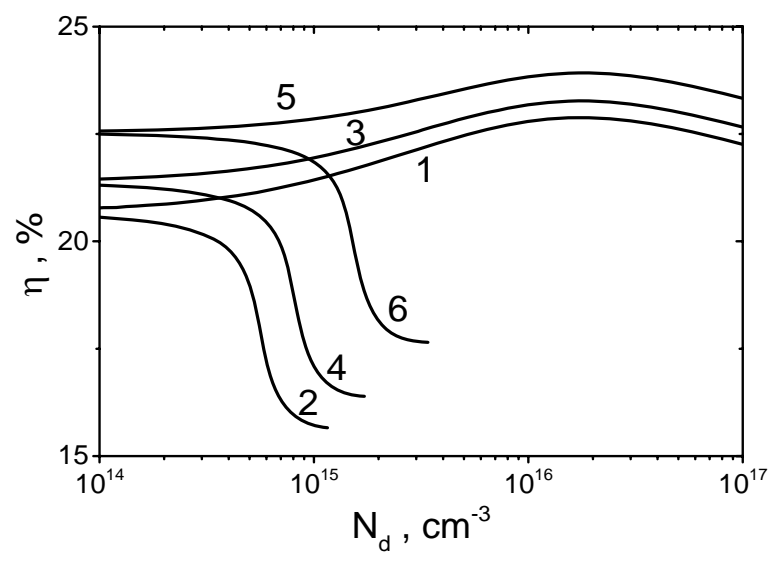

Fig. 6. Photovoltaic conversion efficiency dependence on the base doping level $N_{d}\left(N_{a}\right)$ with different base thicknesses: curves $1,3,5$ are plotted for the $n$-type base, and curves 2, 4, 6 are plotted for the $p$-type base. The following parameters were used in construction of the plot: $J_{S C}=39.5 \mathrm{~mA} / \mathrm{cm}^{2}, S=1 \mathrm{~cm} / \mathrm{s}, R_{S}=$ $0.21 \mathrm{Ohm}$. The base thickness $d$ was equal to $300 \mu \mathrm{m}(1,2)$, $200 \mu \mathrm{m}(3,4), 100 \mu \mathrm{m}(5,6)$. The rest parameters were the same as those used in Fig. 3 curves plotting.

\section{Comparison of the experimental and calculated a-Si:H-n-Si HIT cells parameters' values}

Experimental samples HIT SC were made at Institute for Solar Energy Research Hamelin (ISFH, Germany) with participation of one of this paper authors (M.V. Gerasimenko). The best sample with the efficiency $19.4 \%$, that is very close to the calculated values, was made on (100)-oriented and on textured $\mathrm{Si}$ wafer substrates.

After a-Si:H deposition, we perform quasi-steady state photoconductance decay measurements [12] and obtain the lifetime data equal to $1.4 \mathrm{~ms}$. Short-circuit current $I_{S C}$ was measured under illumination with a flash light while the current-voltage curves are measured under LED-array illumination $(\lambda=950 \mathrm{~nm})$ with an intensity that produces the same $I_{S C}$.

In what follows, let us compare the calculated and experimental characteristics of HIT solar cells with those obtained in the experiment in SC with the base of $n$-type: $J_{S C}=36.03 \mathrm{~mA} / \mathrm{cm}^{2}, V_{O C}=0.703 \mathrm{~V}, F F=0.77$, $\eta=19.4 \%, S_{S C}=3.96 \mathrm{~cm}^{2}$ for the case when the SC base region parameters were equal to the following values: $N_{d} \approx 1.6 \cdot 10^{15} \mathrm{~cm}^{-3}, d \approx 300 \mu \mathrm{m}, \tau_{\mathrm{SR}} \approx 1.4 \mathrm{~ms}$.

HIT solar cell manufacturing technology included operations of cleaning of crystalline silicon wafer surface, surfaces texturing (pyramidal surface relief patterning), acid underetching of the surface layer, putting of optimum thickness layer of intrinsic amorphous hydrogenated silicon $(i) \mathrm{a}-\mathrm{Si}: \mathrm{H}$ on both wafer surfaces. Then, $n-c \mathrm{Si} /\left(n^{+}\right)$a-Si:H isotype heterojunction was formed on the back side surface and $n-c \mathrm{Si} /\left(p^{+}\right)$a-Si:H anisotype heterojunction was formed on the front side surface. The transparent conductive layers of indium and tin oxides mixture were deposited on both surfaces, and then the low-temperature annealing was realized to reduce the series resistance. The contacts deposition was the finishing operation: a solid contact was deposited on the back side and the net-like contact was deposited on the front side. The HIT solar cell area was about $4 \mathrm{~cm}^{2}$. This technology was described in detail in the publication [13].

To fabricate SC, we used $n$-type silicon obtained using zone melting technique. Its resistivity at $T=300 \mathrm{~K}$ was $3 \mathrm{Ohm} \mathrm{cm}$. The doping level, as determined from the capacitance-voltage profiling at inverse bias, is $N_{d}=$ $1.6 \cdot 10^{15} \mathrm{~cm}^{-3}$ with $15 \%$ inaccuracy.

Let us compare next the calculated and experimental characteristics of HIT solar cells with those obtained in the experiment in SC with the base of $n$-type: $I_{S C}=142.7 \mathrm{~mA}, V_{O C}=0.703 \mathrm{~V}, F F=0.77, \eta=19.4 \%$, $S_{S C}=3.96 \mathrm{~cm}^{2}$ for the case when the SC base region parameters were equal to the following values: $N_{d} \approx$ $1.6 \cdot 10^{15} \mathrm{~cm}^{-3}, d \approx 300 \mu \mathrm{m}, \tau_{\mathrm{SR}} \approx 1.4 \mathrm{~ms}$. The shortcircuit current density was $J_{S C}=36.03 \mathrm{~mA} / \mathrm{cm}^{2}$.

Fig. 7 shows the current-voltage curve of SC studied here.

The theoretical current-voltage curve was obtained using the expressions (12) and (13). Its good agreement with the experimental values confirms the correctness of our model.

To calculate $R_{\text {Auger }}$, the relation (8) was used when composing Table 1, and the relation (9) was used when composing Table 2, respectively. The first line of Tables 1 and 2 shows the calculated characteristics of the HIT solar cells and base region parameters obtained as a result of calculation by using formulas given above. As can be seen from a comparison of the calculated and experimental parameters given in the first lines of Tables 1 and 2 , they are identical, if $S=12 \mathrm{~cm} / \mathrm{s}$, and $R_{S}=$ $0.21 \mathrm{Ohm}$. Note that the calculated value of the fillfactor $F F$ for current-voltage characteristic in this case is 0.77 , and it coincides well with the experimental value.

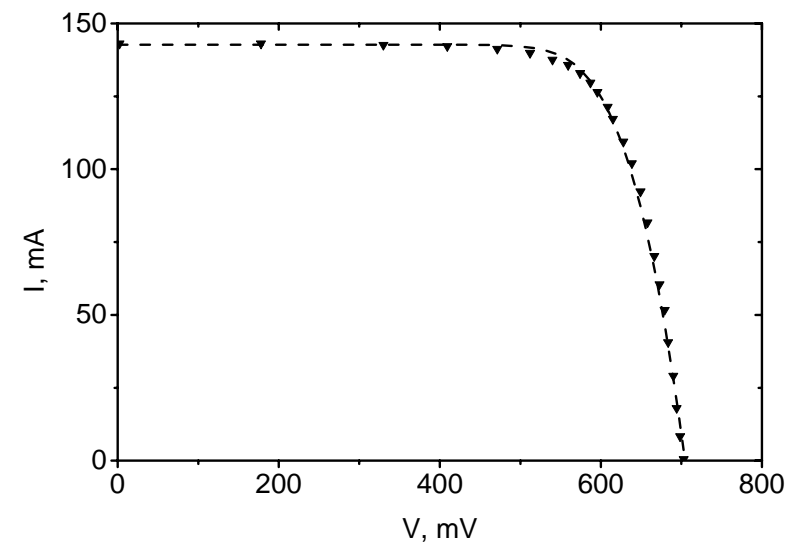

Fig. 7. The experimental (symbols) and theoretical (line) current-voltage curves of an illuminated HIT element. 
Table 1. The HIT SC parameters obtained using (8).

\begin{tabular}{|l|c|c|c|c|c|c|c|c|c|}
\hline \multicolumn{1}{|c|}{ Sample } & $\begin{array}{c}N_{d,} \\
\mathrm{~cm}^{-3}\end{array}$ & $d, \mu \mathrm{m}$ & $\begin{array}{c}\tau_{\mathrm{SRH}}, \\
\mathrm{ms}\end{array}$ & $\begin{array}{c}J_{S C}^{\exp }, \\
\mathrm{mA} / \mathrm{cm}^{2}\end{array}$ & $V_{O C}, \mathrm{mV}$ & $\eta, \%$ & $\mathrm{FF}, \%$ & $\begin{array}{c}S, \\
\mathrm{~cm} / \mathrm{s}\end{array}$ & $\begin{array}{c}R_{S}, \\
\text { Ohm }\end{array}$ \\
\hline$n$-type & $1.6 \cdot 10^{15}$ & 300 & 1.4 & 36.03 & 704 & 19.4 & 76.6 & 12 & 0.21 \\
\hline$n$-type & $1.6 \cdot 10^{15}$ & 300 & 1.4 & 39.5 & 719 & 21.8 & 76.6 & 1 & 0.21 \\
\hline$n$-type & $4 \cdot 10^{16}$ & 300 & 1.4 & 36.03 & 710 & 21.0 & 82.1 & 12 & 0.21 \\
\hline$n$-type & $2 \cdot 10^{16}$ & 300 & 1.4 & 39.5 & 719 & 23.2 & 81.8 & 1 & 0.21 \\
\hline
\end{tabular}

Table 2. The HIT SC parameters obtained using (9).

\begin{tabular}{|c|c|c|c|c|c|c|c|c|c|}
\hline Sample & $\begin{array}{c}N_{d}, \\
\mathrm{~cm}^{-3}\end{array}$ & $d, \mu \mathrm{m}$ & $\begin{array}{c}\tau_{\mathrm{SRH}}, \\
\mathrm{ms}\end{array}$ & $\begin{array}{c}J_{S C}^{\exp }, \\
\mathrm{mA} / \mathrm{cm}^{2}\end{array}$ & $V_{O C}, \mathrm{mV}$ & $\eta, \%$ & $\mathrm{FF}, \%$ & $\begin{array}{c}S, \\
\mathrm{~cm} / \mathrm{s}\end{array}$ & $\begin{array}{c}R_{S}, \\
\text { Ohm }\end{array}$ \\
\hline$n$-type & $1.6 \cdot 10^{15}$ & 300 & 1.4 & 36.03 & 704 & 19.4 & 76.5 & 12 & 0.21 \\
\hline$n$-type & $1.6 \cdot 10^{15}$ & 300 & 1.4 & 39.5 & 719 & 21.7 & 76.5 & 1 & 0.21 \\
\hline$n$-type & $4 \cdot 10^{16}$ & 300 & 1.4 & 36.03 & 709 & 20.7 & 81.0 & 12 & 0.21 \\
\hline$n$-type & $2 \cdot 10^{16}$ & 300 & 1.4 & 39.5 & 719 & 22.9 & 80.8 & 1 & 0.21 \\
\hline
\end{tabular}

For the moderate levels of the base doping $\left(\sim 1.6 \cdot 10^{15} \mathrm{~cm}^{-3}\right)$ the HIT solar cell parameters, given in the second line of Tables 1 and 2, practically coincide. The calculated SC parameters were obtained using the following values: $S=1 \mathrm{~cm} / \mathrm{s}, J_{S C}=39.5 \mathrm{~mA} / \mathrm{cm}^{2}[4,14]$.

Also, the parameters in the third and the fourth rows of the Tables 1 and 2, obtained using (8) and (9), are somewhat different. However, as seen from these tables, the photoconversion efficiency increases with doping level, whereas the parameters obtained using (8) and (9) differ by not more than $1 \%$.

Thus, as seen from our analysis, the base doping level should be optimized in order to achieve the highest efficiency at the fixed values of the remaining parameters.

\section{Conclusions}

The theoretical analysis of high-efficient silicon SC efficiency is carried out depending on the type and level of doping of the base for the case when Fe level induces the $\mathrm{SRH}$ recombination. It has been shown that the photovoltaic conversion efficiency in the case of $n$-type base increases with increasing the base doping level, reaches its maximum and begins to decrease. This decrease is determined by the band-to-band Auger recombination predominance. In the case of SC with the $p$-type base, the photovoltaic conversion efficiency decreases with the base doping level increase. The obtained result is associated with a strong asymmetry of Fe level parameters, due to it the strong decrease in the $\tau_{\mathrm{SRH}}$ value occurs in the $p$-type samples in the field of the doping levels' values range characteristic for the Si solar cells.
The calculated and experimental values of characteristics of HIT SC based on a-Si:H-n-Si are compared. The effective surface recombination velocity and serial resistance values are defined from the comparison; complete coincidence is achieved between theory and practice.

It is shown that the base doping level is one of the most important parameters of the HIT solar cells. This parameter is subject to optimization and the use of its optimal values for SC of the order of $(2-4) \cdot 10^{16} \mathrm{~cm}^{-3}$ with the $n$-type base makes it possible to increase significantly the $\eta$ value.

\section{References}

1. A. Hangleiter, Nonradiative recombination via deep impurity levels in silicon: Experiment // Phys. Rev. B, 35(17), p. 9149-9160 (1987). DOI: 10.1103/PhysRevB.35.9149.

2. L.G. Geerings and D. Macdonald, Base doping and recombination activity of impurities in crystalline silicon solar cells // Progr. Photovolt: Res. Appl. 12(4), p. 309-316 (2004). DOI: 10.1002/pip.546.

3. B.L. Sopori, L. Jastrzebski, T. Tan, A comparison of gettering in single- and multicrystalline silicon for solar cells // 25th IEEE Photovoltaic Specialists Conference, 1996, p. 625-628. DOI: 10.1109/ PVSC.1996.564206.

4. A.V. Sachenko, A.I. Shkrebtyi, R.M. Korkishko, V.P. Kostylyov, N.R. Kulish, I.O. Sokolovskyi, Features of photoconversion in highly efficient silicon solar cells // Semiconductors, 49(2), p. 264269 (2015). DOI: 10.1134/S1063782615020189. 
5. J.S. Blakemore, Semiconductor Statistics. Pergamon Press, Oxford, 1962.

6. A. Richter, S.W. Glunz, F. Werner, J. Schmidt, A. Cuevas, Improved quantitative description of Auger recombination in crystalline silicon // Phys. Rev. B, 86, 165202 (2012). DOI: 10.1103/ PhysRevB.86.165202.

7. A.P. Gorban, A.V. Sachenko, V.P. Kostylyov et al., Effect of excitons on photoconversion efficiency in the $\mathrm{p}^{+}-\mathrm{n}-\mathrm{n}^{+}-$and $\mathrm{n}^{+}-\mathrm{p}-\mathrm{p}^{+}$-structures based on singlecrystalline silicon // Semiconductor Physics, Quantum Electronics and Optoelectronics, 3(3), p. 322-329 (2000).

8. A.V. Sachenko, A.P. Gorban, V.P. Kostylyov, I.O. Sokolovskyi, The radiative recombination coefficient and the internal quantum yield of electroluminescence in silicon // Semiconductors, 40(8), p. 884-889 (2006). DOI: 10.1134/ S1063782606080045.

9. A. Hangleiter, and R. Häcker, Enhancement of band-to-band Auger recombination by electronhole correlations // Phys. Rev. Lett. 65(2), p. 215218 (1990). DOI: 10.1103/PhysRevLett.65.215.
10. A.V. Sachenko, A.P. Gorban, V.P. Kostylyov, I.O. Sokolovskyi, Quadratic recombination in silicon and its influence on the bulk lifetime // Semiconductors, 41(3), p. 281-284 (2007). DOI: $10.1134 / \mathrm{S} 1063782607030074$.

11. M.J. Kerr and A. Cuevas, General parameterization of Auger recombination in crystalline silicon // J. Appl. Phys. 91(4), p. 2473-2480 (2002). DOI: 10.1063/1.1432476.

12. R.A. Sinton, A. Cuevas, and M. Stuckings, Quasisteady-state photoconductance, a new method for solar cell material and device characterization // 25th IEEE Photovoltaic Specialists Conf., 1996, p. 457-460. DOI: 10.1109/PVSC.1996.564042.

13. R. Gogolin, R. Ferre, M. Turcu, N.-P. Harder, Silicon heterojunction solar cells: Influence of $\mathrm{H}_{2}$-dilution on cell performance // Solar Energy Materials \& Solar Cells, 106, p. 47-50 (2012). DOI: 10.1016/j.solmat.2012.06.001.

14. A. Jano, S. Tohoda, K. Matsuyama et al., 24.7 record efficiency hit solar cell on thin silicon wafer // 28th European Photovoltaic Solar Energy Conference and Exhibition, 2013, p. 1846-1848. DOI: 10.4229/28thEUPVSEC2013-2AO.2.5. 